CIC. Cuadernos de Información y Comunicación ISSN: 1135-7791

http://dx.doi.org/10.5209/CIYC.60684

\title{
Los MOOC como herramientas educomunicativas para el desarrollo: Una revisión de las iniciativas de EEUU, Japón y Europa
}

\author{
Francisco Cabezuelo Lorenzo; Juan Luis Manfredi Sánchez
}

Enviado: 13 de marzo de 2018 / Aceptado: 30 de marzo de 2018.

Resumen. Este trabajo propone el uso de la herramienta educativa de los MOOC como una fórmula internacional para romper con las desigualdades educativas y la brecha digital. La metodología se centra en el análisis del estado del arte y la investigación sobre las nuevas herramientas digitales internacionales. Se centra en el estudio de casos y compara las estrategias seguidas por Estados Unidos, la Unión Europea y Japón. Se pretende definir y contextualizar las decisiones tomadas, los riesgos y las oportunidades. La novedad del trabajo consiste en el estudio del fenómeno de la cooperación y de la nueva diplomacia digital y sus implicaciones en la posible reducción de las desigualdades socioeducativas.

Palabras clave: MOOC, Comunicación Internacional, Brecha Digital, Educación, Desigualdad.

[en] MOOC as educomunical tools for development: a revision of the initiatives of the USA, Japan and Europe

\begin{abstract}
This paper proposes the use of MOOCs as an educational tool and international strategy to end the educational inequalities and the digital divide. The methodology focuses on the analysis of academic literature and previous research on the field of the new international digital tools. As well, this paper focuses on the case-study and compared strategies followed by the United States, the European Union and Japan. It tries to define and contextualize their decisions, risks and opportunities. The novelty of the work is the approach to MOOCs envisaged as a tool for the new digital cooperation and diplomacy and its implications for the possible reduction of socio-educational inequalities.

Keywords: MOOC, International Communication, Digital Divide, Education, Inequality.
\end{abstract}

Sumario. 1. Introducción y justificación. 2. Marco teórico y contexto histórico. 3. Metodología. 4. Análisis. 4.1. Las acciones educativas internacionales de las grandes potencias mundiales. 4.2. El nuevo escenario digital de las relaciones internacionales. 4.3. El desarrollo de los MOOC como herramientas educomunicativas contra la desigualdad. 4.4. La estrategia de Estados Unidos, Unión Europea y Japón. 5. Conclusiones. Referencias.

Cómo citar: Cabezuelo Lorenzo, F.; Manfredi Sánchez, J. L. (2018). Los MOOC como herramientas educomunicativas para el desarrollo: Una revisión de las iniciativas de EEUU, Japón y Europa, en CIC. Cuadernos de Información y Comunicación 23, 175-188.

\section{Introducción y justificación}

Vivimos en un contexto global. El fenómeno de la mundialización es hoy toda una realidad. A pesar de estos avances y de estar rodeados de tecnología, existen graves diferencias y desigualdades en el acceso a las oportunidades (Oñate, Fanjul \& Cabe- 
zuelo, 2015). Los estudios demuestran una gran desigualdad en los intercambios comunicativos en los procesos de comunicación social. En la mayoría de ocasiones son problemas ligados a las diferencias socioeconómicas de las audiencias, ya sea por cuestiones de edad, género, alfabetización digital, contenidos especializados de difícil compresión o por cuestiones ligadas a los problemas en la recepción de esos productos mediáticos.

La misma construcción de muchos discursos mediáticos, que se alimenta de estereotipos anclados en el pasado, generan en muchas ocasiones representaciones burlescas, incompletas o estigmatizadoras de la realidad, al tiempo que ignoran, menosprecian o hacen invisibles otras realidades minoritarias, simplemente en muchas ocasiones por cuestiones raciales, económicas, políticas, étnicas, o simplemente geográficas.

\section{Marco teórico y contexto histórico}

El mundo ya no está dividido en dos grandes bloques como en los años de la Guerra Fría y el Telón de Acero. Sin embargo, los conflictos armados siguen azotando el planeta. No son los únicos dramas que vive la sociedad contemporánea. El fomento de la democracia, la libertad y la seguridad, la lucha contra el terrorismo y a favor de la paz, junto con la acuciante necesidad de proteger el medio ambiente y de promover un consumo responsable, son algunos de los nuevos retos de nuestro mundo. Sin embargo, hemos heredado otros problemas del pasado. Hay que seguir luchando para reducir la diferencia y brecha socioeconómica entre los ciudadanos y los países más pobres y más ricos. Esta preocupación viene ya lejos. El problema de la exclusión social ha sido relevante en la literatura académica y política reciente, entre la que destacan tanto los trabajos de la denominada Escuela de Frankfurt como de la escuela británica de Estudios Culturales.

Paralelamente surgían en esos años nuevas iniciativas educativas para romper con la desigualdad. Es el caso de la educación a distancia o educación en abierto. Un par de hitos marcan los inicios de este movimiento que apunta hacia la educación abierta: la creación del International Council for Open and Distance Education (ICDE) en Canadá en 1938, y el inicio de la Open University en el Reino Unido en 1969, tal y como recogen Chiappe-Laverde, Hine y Martínez-Silva (2015: 10). Ante estas nuevas fórmulas de enseñanza-aprendizaje, los docentes de todo el mundo han tenido que ser instruidos y formados en estas nuevas metodologías. Del mismo modo, una gran cantidad de repositorios de estos materiales han sido creados acompañados de igual número de estrategias de divulgación y familiarización entre la comunidad académica (Lehman, 2007). Además, en la primera década del siglo XXI, han aparecido en todo el mundo diferentes iniciativas relacionadas con la apertura en educación, la mayoría de ellas basadas en promover el acceso a recursos educativos abiertos, especialmente centrados en la creación, uso y catalogación de materiales educativos digitales como los objetos de aprendizaje reutilizables (Campbell, 2004). Hay que añadir además que en un principio todas estas actividades han sido construidas bajo la expectativa según la cual esta estrategia traerá beneficios significativos mediante el compartir recursos y experticia entre la comunidad académica, lo cual permitirá generar innovación educativa.

Así, desde la segunda mitad del siglo pasado se han puesto en marcha cientos de iniciativas sociales, políticas, institucionales y diplomáticas de cooperación entre los 
llamados países del primer mundo y los denominados países en vías de desarrollo. Han destacado iniciativas de éxito como los programas Fulbright de Estados Unidos o Erasmus de la Unión Europea. En este contexto, las nuevas formas de cooperación educativa y diplomacia pública han ganado espacio en los recientes estudios de comunicación internacional. La comunicación internacional ha asumido una función fundamental en las relaciones internacionales. Esta nueva forma de relacionarse entre países y gentes se fundamenta en los intercambios culturales. Así, la nueva cooperación consiste en la estrategia de información, educación y entretenimiento que tiene como objetivo los intercambios culturales con públicos extranjeros o internacionales. Es una línea de la acción exterior de muchos gobiernos.

Lo que distingue la nueva cooperación intercultural y la diplomacia pública de la propaganda es el interés en el beneficio mutuo, la cooperación y la transparencia. Por el contrario, la propaganda es coercitiva, impone los contenidos y no abre espacios para el diálogo y el cambio de actitudes. La multiplicación de acciones, sobre todo en el ámbito de la cultura, se legitima como una vía para la promoción de ideas, el desarrollo y la igualdad. Se entiende que la dinámica cultural es más acertada para la transformación de la opinión pública para alcanzar la igualdad entre ciudadanos y naciones. En diplomacia pública, la difusión de las ideas con el objeto de atraer recursos y personas tiene que basarse en la confianza y el entendimiento, no desde la superioridad, sino desde la cooperación. Por eso, los programas educativos son prioritarios en el diseño y la ejecución de las estrategias de cooperación internacional en el siglo XXI.

\section{Metodología}

En este caso, se trata de un trabajo conceptual o teórico que tiene como finalidad mejorar la comprensión por parte de la comunidad académica sobre las diferentes manifestaciones de la desigualdad en el plano de los nuevos medios y herramientas de comunicación digitales ligados a la educación, que es la única y la más poderosa herramienta para acabar con las desigualdades.

En primer lugar, se revisa la literatura sobre los programas educativos internacionales y la educación en abierto o a distancia. Para ello, se adoptó el método de revisión por integración de Whittemore \& Knafl (2005), que incluye la consulta por separado de dos procesos separados pero estrechamente relacionados como son la revisión de literatura y el análisis de datos. Esta metodología implica la creación de un marco teórico propio para la revisión, en una aproximación que se fundamenta en una apropiada selección de bases de datos, el establecimiento de criterios para la selección y rechazo de otros textos, y que termina con un proceso de reducción de los documentos y una fase de relectura final.

Partiendo de la primera literatura académica existente sobre este tema gracias autores como Barth, (1972) y Walberg \& Thomas (1972), se llega a planteamientos más actualizados y contemporáneos como los de D'Antoni (2009) que reflexionan sobre el actual movimiento educativo en abierto, sin olvidar autores intermedios que se centraron en el estudio de los principios e influencias globales del movimiento de software libre de finales de los años setenta y ochenta del pasado siglo.

Se continúa con la definición de los elementos esenciales de la nueva cooperación educativa digital para ver en qué aspectos se ha avanzado más a la luz de la transfor- 
mación tecnológica. Se analiza cómo la educación y la comunicación internacional se han insertado en los flujos de los intercambios internacionales y cómo las tecnologías han acelerado determinados procesos socioeducativos. Entre otros factores, la acelerada mediatización de la esfera pública y la difusión de nuevas tecnologías de información en los últimos años hacen necesario revisitar lo que sabemos en relación a este tema.

Desde el respecto a todos los puntos de vista teóricos, ideológicos y pedagógicos, este trabajo propone el uso de la herramienta educativa de los MOOC como una fórmula internacional y transnacional para romper con las desigualdades educativas y la brecha digital. Desde una perspectiva iberoamericana, este artículo quiere presentar nuevas perspectivas entorno a los MOOC. Conforme a los expuestos por Awad, Domínguez y Bulnes (2013: 31) este trabajo también parte del punto de partida crítico frente a la tecnología y también estima que la narrativa sobre la transformación de las audiencias en la era de las tecnologías digitales tiene dos problemas. Por un lado, se puede caer en el determinismo tecnológico, al suponer una transformación casi automática de las audiencias de pasivas a activas gracias a las nuevas tecnologías. Y en segundo lugar, tampoco se puede caer en el error de pensar que la interacción a través de las nuevas fórmulas digitales de comunicación es igualmente relevante en términos de participación mediática.

Metodológicamente, este trabajo parte del análisis del estado del arte y la investigación sobre las nuevas herramientas digitales internacionales, seleccionando aquellas como los MOOC que son dignas de convertirse en objeto de estudio por parte de los académicos preocupados en cuestiones ligadas a la comunicación y desigualdad. Seguidamente, se centra en el estudio de casos y compara las estrategias seguidas por Estados Unidos, la Unión Europea y Japón. Se pretende definir y contextualizar las decisiones tomadas, los riesgos y las oportunidades. La novedad del trabajo consiste en el estudio del fenómeno de la cooperación y de la nueva diplomacia digital y sus implicaciones en la posible reducción de las desigualdades socioeducativas.

\section{Análisis}

Muchas estrategias de cooperación internacional y de diplomacia pública requieren de una política cultural organizada, articulada de modo que puede influir en la arena internacional. La comunicación, en un sentido muy amplio, y la educación son las vías de acción preferente para lograr nuevos puentes educomunicativos contra la desigualdad. La primera tiene como función mediar la experiencia con amplias capas de población. El cine, el audiovisual, la literatura o la música se emplean para incrementar la notoriedad y el reconocimiento de un país, fomentar el turismo generalista o los viajes de estudio, así como apoyar la comercialización de productos y servicios en mercados exteriores. Los nuevos programas de cooperación intercultural y educativa financian la participación en ferias y festivales, las traducciones, la creación de espacios preferentes en las bibliotecas (American Corners antes y American Spaces ahora, por ejemplo), las emisiones internacionales o cofinancia proyectos de alguna naturaleza (el programa europeo audiovisual Media o Ibermedia con modelos recurrentes). La apuesta por contenidos audiovisuales se explica por su facilidad para transportar emociones y valores. 
En el mundo de las relaciones internacionales, la educación plantea nuevos objetivos y, en consecuencia, requiere otras estrategias. Los programas actúan en el medio plazo y son individualizados al máximo. Cada alumno elige el programa, la universidad o el destino. Se persigue que la experiencia sea única, aunque se enmarque dentro de las acciones destinadas a colectivos académicos, periodísticos o de otra naturaleza (Manheim, 1994). Esta misión está presente en la legislación fundacional de las políticas educativas internacional estadounidense. En 1948 se aprueba la Information and Educational Exchange Act que sostiene que "el intercambio de personas y proyectos constituye un técnica integral y esencial en la consecución de los objetivos generales del programa de intercambio educativo". Del mismo modo, estima que "a través de la experiencia personal y la influencia se promueve un mejor entendimiento de Estados Unidos fuera de nuestras fronteras", tal y como recoge Espinosa (1975: 342).

Los programas educativos pues centran la actividad en el ámbito más personal y el compromiso a través de acciones educativas o científicas. La capacidad de influencia se concentra en el individuo, quien pertenece a algún tipo de elite en el país natal. La personalización facilita el diseño de mensajes. Los programas no pueden excluir por razón económica, social o de origen, pero sí que identifican personas con alto potencial para influir en un entorno concreto. Se persigue conectar futuras clases medias mediante un contacto personal, esto es, la creación de redes profesionales que contribuyan al desarrollo de proyectos concretos en el campo de la tecnología, la innovación, la ciencia y el I+D. En suma, estos programas fijan las bases de la construcción de relaciones personales, individuales. Por eso, consumen muchos recursos económicos, requieren una acción consistente y son arriesgados. El diseño del programa, la selección de los candidatos o la elección de un programa de formación relevante son elementos inestables. Cualesquiera que sea la naturaleza del programa (becas, intercambios, formación, conferencias, seminarios, redes sociales profesionales) no exime de tales condicionamientos.

\subsection{Las acciones educativas internacionales de las grandes potencias mundiales}

Los fundamentos y enfoques anteriormente citados son la base el desarrollo de los programas educativos por potencias económicas del primer mundo. La proyección exterior de la identidad sigue las pautas culturales, con diferencias manifiestas en el ámbito de los valores. Esto significa que el lanzamiento y la dotación de recursos para la creación de nuevos contenidos educativos persiguen objetivos políticos diferentes y, por ello, se inciden en unos u otros programas.

En Estados Unidos, a pesar de la deriva proteccionista del nuevo presidente Donald Trump, los programas educativos gozan de una innegable dimensión internacional. Se sistematizan en este principio tras la aprobación allá por 1961 de la Mutual Educational and Cultural Exchange Act (US Code - Título 22: Foreign Relations and Intercourse - Chapter 33) que se conoce como la Fulbright-Hays Act.

La sistematización de los intercambios y la educación internacional se explica como respuesta a la ofensiva comunista. Ante el crecimiento y el éxito de la propaganda en Europa, se decide organizar la cooperación educativa con el ánimo de vincular la inversión económica al desarrollo educativo y transformar la visión que tenían determinados países acerca de Estados Unidos.

Por su parte, en Europa se puso en marcha el exitoso programa Erasmus, que es el epítome de los intercambios, y que nació con el objetivo de atender a las necesi- 
dades de enseñanza y aprendizaje de todos los participantes en educación superior formal y en formación profesional de grado superior, cualquiera que sea la duración de la carrera o cualificación, incluidos los estudios de doctorado, así como a las instituciones que imparten este tipo de formación, tal y como se explica en la web oficial del Organismo Autónomo de Programas Educativos Europeos (OAPEE).

Destaca el nuevo programa Erasmus Plus que abarca el período comprendido entre los años 2014 y 2020 y forma parte de la estrategia Educación y Formación del programa Horizonte 2020. La Comisión Europea establece que el programa tiene dos objetivos concretos. El primero es el apoyo a la realización de un espacio europeo de educación superior (EEES) y el segundo es que la educación superior contribuya al proceso de innovación. Con tales fines, la Comisión Europea tiene previsto destinar 4.920 millones de euros para dicho sexenio. El OAPEE estima que tres millones de personas se han beneficiado de algún tipo de acción Erasmus desde su creación en 1987.

Por su parte, The Japan Exchange \& Teaching Program (JET) se comenzó también en 1987. Su objetivo es el apoyo a la internacionalización del país y la promoción del entendimiento mutuo con terceros países mediante los programas de intercambio. Estos se dirigen a la población joven, que son invitados a trabajar en instituciones de naturaleza pública y centros de enseñanza durante un año. El programa JET es una actividad financiada por los ministerios de Asuntos Exteriores, Educación y los gobiernos locales a través del Consejo de Autoridades Locales para las Relaciones Internacionales. En 2013, se beneficiaron 4.372 participantes. Estados Unidos lidera el programa con 2.359 personas, seguido de Canadá (484), Reino Unido (388), Australia (2003) y Nueva Zelanda (255).

\subsection{El nuevo escenario digital de las relaciones internacionales}

El último factor es el crecimiento de los medios digitales y la expansión del entorno digital. Redes sociales, plataformas digitales, dispositivos móviles, tabletas, la mensajería instantánea o los periódicos digitales han creado un nuevo ecosistema de relaciones informativas, políticas y ciudadanas (Castells, 2008: 13). Interesa como elemento transformador de la diplomacia pública en la medida que el entorno digital se convierte en un ámbito de discusión, de influencia, de generación de prestigio y de desarrollo de facultades. Muchas de las nuevas formas de comunicación internacional no admiten fronteras físicas, lo que hace más difícil que se pueda ser bloqueado o censurado. Es un entorno esencial para el empoderamiento de nuevas generaciones de ciudadanos.

Mención especial merece el desafío demográfico para las nuevas estrategias de diplomacia pública. Alrededor del 45\% de la población mundial tiene menos de 24 años, según datos publicados por la Population Reference Bureau en su última edición. Y es una tendencia en crecimiento. Las redes son ahora, y lo serán en los próximos años aún más, un entorno necesario para la difusión de los mensajes, para la gestión de la influencia y para el desarrollo de programas educativos.

Los nuevos medios y las redes sociales son transformadores de la cooperación internacional y de las estrategias que se siguen. Los principios de la nueva cooperación internacional basada en la diplomacia digital son la flexibilidad, la escalabilidad y la supervivencia, ejes de la sociedad red (Castells, 2009: 23). La literatura académica (Heine, 2008: 271 y Gstöhl, 2012: 49) ya ha acuñado la expresión de “diploma- 
cia en red", que explica cómo la multiplicidad de fuentes de legitimación, participación y comunicación han entrado de lleno en la arena internacional y en el ámbito específico de las relaciones internacionales. Se caracteriza por su naturaleza abierta y por la necesidad de abrir los circuitos convencionales y abrirse a nuevos otros actores como organizaciones no gubernamentales, universidades, estudiantes, activistas y blogueros.

\subsection{El desarrollo de los MOOC como herramientas educomunicativas contra la desigualdad}

En el ámbito de la cooperación y diplomacia pública, cada país ha diseñado metodologías distintas para abrazar la digitalización. En el ámbito digital, las estrategias digitales concuerdan con las afinidades culturales y los valores de la política exterior que se han desarrollado bajo el paraguas de la política cultural exterior. Así, los MOOC han emergido como una herramienta de creciente demanda en este proceso de innovación y transformación de la diplomacia pública.

La palabra MOOC viene del acrónimo anglosajón Massive Open Online Course. Los MOOC representan la cara más visible de la aplicación de la innovación tecnológica al ámbito educativo. Son la variante más conocida, aunque existen otras fórmulas de recursos digitales y tecnología. Son el epítome de los personal learning environment, que mezclan las herramientas de aprendizaje de forma flexible, crean una digital communities of practice y exponen sus contenidos en abierto.

Un MOOC consiste en la práctica en un conjunto de videos online, lecturas, participación en foros y tests para controlar la evolución del aprendizaje. Pero no es un curso online al uso: tiene que dotarse de un entorno ágil, adaptar la oferta a un entorno cambiante, ser de naturaleza internacional y abierta, vincular el proyecto con la comunidad de aprendizaje, utilizar las redes sociales, estar abierto a las necesidades de los alumnos y contar con una presencia masiva de alumnos.

Los MOOC suponen una innovación en el aprendizaje (la metodología y la pedagogía del curso) y en la educación (tipo de oferta, diseño del curso, número de alumnos al mismo tiempo). Es una innovación en la medida que resuelve el problema de la educación masiva y a distancia (accesibilidad a la educación de calidad), al tiempo que crea capital social en torno a la plataforma (eficiencia de los recursos). La oferta educativa ha dejado de ser un bien escaso.

El valor añadido de los MOOC es fuente de discusión. No existe un criterio único ni tampoco las instituciones promotoras se ponen de acuerdo en el objetivo que persiguen. El valor añadido se divide en tres áreas. En relación con las posibilidades de encontrar un empleo, el alumno mejora su marca personal y su capital social porque conecta con personas interesadas en áreas de conocimiento similares a la suya. El empleador puede acceder a estos expedientes e identificar talento. Asimismo, ofrece una especialización diferente a los cursos de posgrado al uso. En el ámbito de la certificación, el alumno puede crear su propia formación de posgrado si abona ciertos servicios. Un alumno puede certificar y verificar que ha cursado determinados cursos, que ha asistido a las clases presenciales o a las tutorías. En tercer lugar, genera valor para las universidades porque reduce los costes de externalización de talleres, seminarios y otros cursos de libre elección.

El MOOC reúne las características antes señaladas como necesarias para crear una estrategia de diplomacia pública en la medida que los cursos pueden servir como 
fuente de escucha de las demandas de la sociedad civil (qué dicen los participantes, tono de la conversación, temas elegidos, prioridades), la promoción de unos intereses concretos (fomento de valores, herramientas o áreas de conocimiento), la diplomacia cultural (la promoción de la lengua y la cultura), los intercambios (los cursos ofrecen interacción con individuos concretos, algunos de ellos de reconocido prestigio profesional o científico) y las emisiones de contenidos audiovisuales (los propios videos, los podcasts).

Por estas razones, Estados Unidos, Japón y la Unión Europea han diseñado cursos digitales. Las estrategias responden a los intereses nacionales y sus respectivos modelos de diplomacia pública. Al mismo tiempo, muestran las limitaciones de recursos (ideas, personas, financiación) y de capacidad de impacto (idioma, cultura). Los MOOC refuerzan la hipótesis de las afinidades culturales que refuerzan los subsistemas internacionales y se apalancan sobre factores políticos y estratégicos.

\subsection{La estrategia de Estados Unidos, Unión Europea y Japón}

Estados Unidos mantiene una larga tradición de diplomacia cultural a través de la ciencia y la tecnología. Internet y la cultura digital ha continuado esta relación, en la línea de otras olas de innovación. El interés particular en la tecnología consiste en la capacidad de crear nuevos mercados (comercio electrónico de bienes y servicios), generar empleo (starts-ups), promover valores (libertad de expresión) y expandir el comercio internacional. La tecnología vehícula cooperación en tanto que transmite estas capacidades y no es, de ninguna forma, neutral.

La expansión del modelo tecnológico de matriz estadounidense requiere la vinculación entre los intereses públicos y privados. Al origen público (de fondos públicos) de Internet se le tiene que sumar la ingente inversión de actores privados que han canalizado la innovación hacia su orientación comercial. Estas operaciones públicoprivadas requieren colaboración, confianza y redes de trabajo extensas. Esta idea de tecnología no neutral se cimenta en que cada decisión apoya u omite determinados valores. La construcción del common good es resultado de la negociación entre interés público e interés privado.

Los MOOC son el epítome de la denominada technology-driven diplomacy and cooperation, según las autoridades estadounidenses. Los jóvenes que emprenden start-ups, medios digitales y otros negocios de base tecnológica son los destinatarios de acciones, programas y cursos de formación. Richard Stengel, actual subsecretario de Relaciones Exteriores del Gobierno del presidente Obama, ha mencionado expresamente la necesidad de vincular la cooperación y la diplomacia digital con la innovación, la economía, la inversión y la internacionalización. Stengel estima que los medios sociales y las tecnologías móviles son "herramientas transformacionales" (2014). Anteriormente, su antecesora Tara Sonenshine, también explicaba que la cooperación y la diplomacia digital sirve a los intereses de política exterior porque los MOOC promueven la interacción con un público global, abre las puertas a la economía de la globalización, fomenta el emprendimiento y apuesta por la innovación. Sonenshine (2014) anunció que Estados Unidos invertiría alrededor de 2.000 millones de dólares en la creación de un sistema educativo digital abierto y en recursos para la formación y la mejora de la empleabilidad global en los próximos años.

Estas iniciativas se han concretado en los MOOC Camps promovidos por el área de Educación y Cultura del Departamento de Estado del Gobierno Obama que ha 
promovido los cursos ligados a la ciencia y tecnología, emprendimiento, y el uso del inglés como lengua extranjera, entre otros. El primer socio tecnológico elegido ha sido Coursera, el consorcio integrado por Princeton University, Stanford University, University of California (Berkeley), University of Michigan-Ann Arbor y University of Pennsylvania, entre otras. Posteriormente, se han ampliado las alianzas, incluyéndola empresa norteamericana $e d X$.

Muchos de estos programas han ido dirigidos a la creación de "nodos de aprendizaje" (hubs), que faciliten la interacción presencial. Se dirigen a una audiencia masiva, pero integran la red ya constituida de sedes e instituciones de la cooperación y diplomacia convencional con las redes y la tecnología. Los becarios Fulbright desempeñan un rol esencial en el éxito de los MOOC porque lideran las conversaciones en los foros y se ocupan de captar talento en la red. Por su parte, las instituciones académicas facilitan a cada participante un encuentro con el responsable de programas educativos para la captación de estudiantes que quieran completar sus estudios en el país (EducationUSA). Las embajadas de Estados Unidos en Armenia, Belice, Benin, Bermuda, China, República Checa, Egipto, India, Indonesia, Iraq, Kenya, Lituania, Macedonia, Madagascar, México, Nigeria, Perú, Rusia, Eslovenia, España, Tanzania y Uruguay usan exitosamente estos programas de cooperación educativa. Estas actividades están en sintonía con el impulso de la actividad emprendedora a nivel global. Instituciones de naturaleza privada han invertido dinero y generado oportunidades para los cursos de emprendimiento de base tecnológica. Destacan acciones como la Global Entrepreneurship Week que cuenta con el apoyo de Kauffamn Foundation y del World Economic Forum para la promoción del emprendimiento y de las empresas de base tecnológica. Esta acción patrocina un MOOC titulado "Beyond Silicon Valley: Growing Entrepreneurship in Transitioning Economies", que es el que mejor refleja este conjunto de tecnologías, valores e ideas llamadas a cambiar el entorno que les rodea.

A su vez, esta colaboración de dinero público y privado es propia de la expansión de la cooperación y de la diplomacia pública norteamericana. Durante la Guerra Fría, las fundaciones, la filantropía y otros actores no gubernamentales pero de alcance global participaron en la estrategia global de diplomacia pública. Precisamente su carácter no oficial permitía el impulso de determinados valores asociados a la libertad, el libre mercado y la libre circulación de las ideas, es decir, al sueño americano. Por ese motivo, rápidamente han aparecido voces críticas con el proyecto MOOC Camps. Siguiendo la retórica de los estudios culturales, los MOOC son señalados como parte del imperialismo cultural de matriz estadounidense. El modelo educativo, las temáticas y los valores proyectados responden a los intereses de la política exterior estadounidense, copatrocinadores de los cursos. Esta idea de aculturación a través de la cultura es propia de la literatura colonial y anticolonial.

Una muestra de esta relación directa entre diplomacia digital e interés nacional convencional es la decisión de aplicar las sanciones a los propios MOOC. Comoquiera que están prohibidas determinadas exportaciones a Irán, Cuba, Sudán y Siria, los alumnos matriculados en los cursos digitales han sido excluidos, lo que muestra una de las grandes debilidades de estos programas que privan a algunos candidatos. Esta dinámica confirma la idea de que los cursos forman parte de la exportación de servicios educativos, sujetos a sanciones, y no a servicios de naturaleza informativa, cuya internacionalización es más flexible.

La segunda línea crítica consiste en que por su naturaleza digital los MOOC se dirigen de nuevo a una elite de la población; los cursos son gratuitos y abiertos, pero 
los medios tecnológicos para participar en ellos no, lo que ahonda la distancia entre quienes pueden costearse el acceso a Internet y los nuevos medios y quienes no cuentan con esos recursos.

Por su parte, en el ámbito de la Unión Europea (UE), la transformación tecnológica en materia de diplomacia pública no han calado de igual modo. No existe en el acervo comunitario una aproximación tan clara y tan edificante de la acción diplomática en materia de tecnologías. De momento, los avances en materia de tecnología están relacionados con la educación (alfabetización mediática) y las telecomunicaciones (roaming). No hay contenidos específicos relacionados con la libertad de expresión o la participación política. Tampoco hay un proyecto claro de cómo actualizar el programa Erasmus Plus al nuevo contexto de la digitalización.

No obstante, se han promovido al menos dos grandes iniciativas que vinculan el europeísmo, el emprendimiento y los valores europeos. El primer proyecto de impacto es OpenupEd.Eu, lanzado en 2013, coordinado por la European Association of Distance Teaching Universities con el apoyo de once socios procedentes de Francia, Italia, Lituania, Países Bajos, Portugal, Eslovaquia, España, Reino Unido, Rusia, Turquía e Israel. La Comisaria de Educación. Androulla Vassiliou, consideró en su momento que el proyecto reflejaba "determinados valores europeos, como la equidad, la calidad y la diversidad" y que será un "elemento decisivo de la estrategia de Apertura de la Educación” (Comisión Europea, 2014a). El segundo proyecto relevante es Start-up Europe MOOC, que tiene como objetivo el impulso del talento y las habilidades digitales en el continente. Se pretende transforma el entorno económico para el fomento de la innovación, el crecimiento económico y la creación de empleo. Forma parte del Plan de Acción Emprendimiento 2020 y la Agenda Digital (Comisión Europea, 2014b).

En el caso europeo, se observa una estrategia dispersa e irregular. No existe una coordinación interna en la propia Comisión Europea, ya que se publican contenidos sin cierto orden aparente. En relación con los contenidos de los MOOC, podemos indicar que no han sabido aprovechar el patrimonio histórico y cultural de los países europeos. La apuesta por el emprendimiento y las habilidades digitales no es diferencial que diferencie entre plataformas y segmente entre usuarios. En suma, la ausencia de una estrategia avanzada para el impulso de los MOOC con sello europeo es una muestra más de las carencias de la diplomacia pública europea. Se enfrenta al doble reto de los públicos internos (los propios estados miembros) y los públicos externos (otros países): ¿A quién habría que dirigir los cursos para la promoción de la identidad europea? ¿Deben primar las relaciones entre Estados miembros o los terceros países? Las dificultades propias del diseño organizativo y la atribución de competencias impiden el avance rápido en materia educativa. Esta concatenación de problemas parece dificultar el diseño y la ejecución de una estrategia de diplomacia pública digital.

Japón también se ha sumado a la ola MOOC para la consecución de objetivos de política exterior a través del Japan Open Online Education Promotion Council (JMOOC), una institución creada en 2013 cuya misión es la contribución al aprendizaje continuo de toda la sociedad, la promoción de la innovación en las metodologías educativas. Otros elementos indicados en la misión es el reconocimiento social de la certificación educativa digital, (JMOOC, 2014). Señala específicamente que quiere crear MOOC al estilo japonés y no estadounidense, al tiempo que se quiere favorecer la colaboración entre la industria y la academia. Se ha recogido la experiencia de los 
Japanese Open Course Ware, que ya reúne a 41 instituciones de educación superior y ofrece alrededor de 3.000 cursos cada año. Se pretende cubrir el nicho de los cursos MOOC, ya que hay poca oferta en japonés o bien realizada por universidades niponas. El socio tecnológico de referencia es Schoo (http://schoo.jp), una start-up creada en 2013.

Las instituciones japonesas son conscientes de la debilidad de la actividad educativa, sobre todo en áreas que no sean tecnológicas o ingenierías. Esa "impredictabilidad" reduce las posibilidades de éxito, por lo que "el profesorado que imparte lengua y cultura japonesa debería ver los MOOC como una vía para llegar a más alumnos" (McMorran, 2013). Las voces críticas también existen en la sociedad japonesa. Por un lado, la propia legislación nacional dificulta la creación de nuevos contenidos y el uso libre de contenidos de terceros, aunque sea con una finalidad educativa (McLelland, 2013). Otros autores señalan que los MOOC tienden a la espectacularización de la educación superior, que puede minar la calidad de la misma. Otake (2010) recoge el caso de Michael Sandel, profesor de derecho de Harvard cuya popularidad se ha disparado tras la impartición de un MOOC en la plataforma $e d X$.

La estrategia japonesa también demuestra serias limitaciones en materia de política cultural aislada de la práctica política. No basta con una economía fuerte e internacionalizada ni tampoco con una propuesta de valor cultural, sino que es necesario establecer conexiones sociales. La cultura japonesa muestra una capacidad limitada de participación en el intercambio de significados, la circulación de las ideas y la transmisión de iconos globales, textos o representaciones. No es una cuestión de calidad, sino de acceso. Es interesante este caso para el estudio en la medida que desmitifica la capacidad de los MOOC para crear cualidades o agregar valor a una estrategia si no existe una determinación política.

\section{Conclusiones}

Los MOOC son una posible fuente de innovación en la comunicación política internacional. En ámbito de la lucha contra el desempleo y el paro, el emprendimiento y los nuevos negocios, los cursos pueden fomentar la interacción entre ciudadanos, eliminar intermediarios en los procesos de localización socios o aliados internacionales, pueden visibilizar currículos o germinar ideas. En relación con el aprendizaje continuo, los cursos pueden certificar conocimiento, facilitar la adquisición de nuevas habilidades sin necesidad de desplazarse, dotar de una primera experiencia o contacto con un país extranjero, identificar universidades o centros de prestigio. En cuanto a la cooperación y a la diplomacia cultural, en sentido amplio, los MOOC son ideales para externalizar talleres, seminarios y todo tipo de actividades ciudadanas. Estas características hacen que los MOOC puedan y deban ser considerados como una herramienta transnacional para acabar con las desigualdades.

Todavía está por ver el nivel de inteligencia que se puede obtener del análisis los big data resultante del agregado de alumnos, cursos, preferencias temáticas, prevención de riesgos, entre otras innovaciones. La emergencia de los MOOC en la cooperación educativa es un hecho cierto. Con distintos objetivos y medios, Estados Unidos, la Unión Europea y Japón se han implicado en la creación, la producción y la difusión de contenidos educativos a través de este tipo de plataformas. 
En el ámbito estratégico, el MOOC se ha integrado en el conjunto de elementos de las políticas culturales y educativas. La afinidad cultural se extiende hacia el ámbito de la ciencia, la tecnología y los valores. Por eso, los tres actores han apostado por un tipo de MOOC afín a sus intereses.

Por su naturaleza tecnológica, los MOOC encajan con las nuevas estrategias de cooperación digital. En apenas tres años, han impactado en la agenda de los tomadores de decisiones y los responsables políticos. Aún no existe una pauta de buenas prácticas y se desconoce cómo evaluar los resultados. Pamment (2014: 58) ha escrito sobre la dificultad de evaluar de acuerdo con elementos racionales. En relación con los contenidos, éstos pueden orientarse hacia valores de lucha contra la desigualdad. De momento, a pesar de la deriva proteccionista del nuevo presidente Donald Trump, parece que de momento solo Estados Unidos tiene claro cómo trabajar en esta línea mediante la creación de títulos propios y la búsqueda de alianzas sólidas con actores globales.

Por su parte, la Unión Europea padece el dilema del doble mercado. El destinatario de los cursos es indeterminado, esto es, no está claro si se dirige a ciudadanos de la UE o bien quiere influir en una audiencia fuera de Europa. Japón quiere utilizar los MOOC como palanca de cambio en los programas educativos y favorecer la internacionalización de los estudios japoneses. Hasta el momento, su cultura y lengua permanece como nicho y se persigue ampliar el número de profesores, estudiantes y profesionales interesados. Cada modelo pretende objetivos diferenciados empleando los MOOC como una suerte de marketing de contenidos.

El análisis de los destinatarios ofrece un resultado interesante. Los programas de intercambio son por definición limitados en su número y alcance. Se dirigen a las elites de un determinado círculo (económico, intelectual o social) y ofrecen una experiencia interactuada. En cambio, los MOOC se plantean como un instrumento de impacto masivo. Un criterio de evaluación podría ser precisamente el número de matriculados o la diversidad geográfica de los mismos. No obstante, resta un análisis cualitativo que permita la medición del impacto de los MOOC en una sociedad foránea, el grado y la calidad de relaciones establecidas entre los participantes y los profesores o la conversión de los matriculados en futuros influyentes en sus sociedades locales.

Entre los puntos críticos, está el auge y la caída de las tendencias tecnológicas. Si el año 2012 fue bautizado como el año de los MOOC (Pappano, 2012), la constante evolución nos lleva a plantearnos qué futuro tienen estos cursos o qué formas tendrán. No hay duda del impacto en los programas de intercambio y en la cooperación internacional, pero falta aún saber cómo se desarrollarán a medio plazo, qué acuerdos se tomarán con otros grandes actores tecnológicos como puedan ser las grandes empresas como Amazon, Apple, Pearson o McGrawHill.

Otra de las críticas recurrentes es la gobernanza de los programas MOOC apoyados por los gobiernos con el ánimo de impulsar la política exterior. Hay intereses contrapuestos, por ejemplo, en qué empresas o modelos de negocio se estudian. Hay que analizar cómo la promoción de ciertos valores, actitudes o comportamientos favorece o perjudica los intereses de los países sin convertir los cursos en propaganda. Por último, habrá que terminar con la denominación masiva de MOOC. No todos los cursos son iguales ni persiguen los mismos fines. Pronto se verá si los MOOC siguen un modelo de educación digital para las elites o para el público generalista, si se especializan por áreas o contenidos, si se centran en público y desarrollo local o bien si se orientan hacia el aprendizaje permanente. 
Como conclusión última, es preciso reivindicar el uso de la herramienta educativa de los MOOC como una fórmula internacional y transnacional para romper con las desigualdades educativas y la brecha digital, ya que sólo la educación puede cambiar el rumbo de las personas y los pueblos.

\section{Bibliografía y fuentes}

Advisory Committee on Cultural Diplomacy (2005). Cultural Diplomacy. The Linchpin of Public Diplomacy, 17-3-2014, p. 7. http://www.state.gov/documents/organization/54374. pdf

Aguaded, I. (2013). The MOOC Revolution: A New Form of Education from the Technological Paradigm? Comunicar, 21(41), 07-08. (DOI: http://doi.org/tnh).

Awad, I. Domínguez, M. y Bulnes, A. (2013). Dirigentas de campamento y actividad mediática: más allá de internet. Cuadernos.info, 33, 29-42. DOI: 10.7764/cdi.33.534

Barth, R. S. (1972). Open Education and the American School. New York: Agathon Press, Inc.

Campbell, L. (2004). Engaging with the Learning Object Economy. In A. Littlehorn (Ed.), Reusing online resources: A Sustainable Approach to E-learning (pp. 35-45). London: Routledge. (http://goo.gl/303GCK) (16-04-2014).

Castells, M. (2008). Comunicación, poder y contrapoder en la sociedad red (I). Los medios y la política. TELOS: Cuadernos de comunicación e innovación, 74, pp.13-24.

Castells, M. (2009). Communication Power, Oxford University Press, Oxford, p.23.

Chiappe-Laverde, Hine y Martínez-Silva (2015). Literature and Practice: A Critical Review of MOOCs. Revista Comunicar, 44. Vol. 22. http://dx.doi.org/10.3916/C44-2015-01

Comisión Europea (2014a): Vassiliou acoge con satisfacción el lanzamiento de los primeros MOOC a nivel universitario. (Noticia del 18-3-2014). http://europa.eu/rapid/press-release_IP-13-349_es.htm

Comisión Europea (2014b): About Start up Europe. (Noticia del 18-3-2014) https://ec.europa.eu/digital-agenda/about-startup-europe

D'Antoni, S. (2009). Open Educational Resources: Reviewing Initiatives and Issues. Open Learning: The Journal of Open, Distance and e-Learning, 24(1), 3-10. (DOI: http://doi. org/fwfdc2).

Espinosa, J. M. (1975). Inter-American Beginnings of U.S. Cultural Diplomacy, 1936-1948. Department of State Washington, D.C., p.342.

González Oñate. C., Fanjul-Peyró, C. \& Cabezuelo-Lorenzo, F. (2015). Use, Consumption and Knowledge of New Technologies by Elderly People in France, UK and Spain. Revista Comunicar. No 45. DOI: 10.3916/C45-2015-02

Gstöhl, S. (2012). Diplomacy in the 21st Century Is Network Diplomacy, Crossroads, III (2), pp. $49-58$.

Heine, J. (2008). On the Manner of Practising the New Diplomacy. En Cooper, A., Hocking, B. \& Maley, W. (Eds.): Global Governance and Diplomacy: Worlds Apart? Palgrave Macmillan, Baskingstoke (EEUU), pp. 271-287.

Institute for Public Policy Research (2013). An avalanche is coming: Higher education and the revolution ahead. Noticia del 18-3-2014. Disponible en la web: http://www.ippr.org/ images/media/files/publication/2013/04/avalanche-is-coming_Mar2013_10432.pdf

Japan Exchange \& Teaching Program (2014). Welcome. Noticia del 18-3-2014. Disponible en la web: http://www.jetprogramme.org/e/introduction/statistics.html 
Lehman, R. (2007). Learning Object Repositories. New Directions for Adult and Continuing Education, 113, 57-66. (DOI: http://doi.org/dfx2fb).

Manheim, J. B. (1994). Strategic Public Diplomacy and American Foreign Policy: the Evolution of the Influence. Oxford University Press: New York.

McLelland, M. (2014). Ethical and legal issues in teaching about Japanese popular culture to undergraduate students in Australia, Electronic journal of contemporary japanese studies, 13 (2).

McMorran, C. (2013). Teaching Japanese Popular Culture in the MOOC World. Possibilities and Challenges, Electronic journal of contemporary japanese studies, 13(2).

Otake, T. (2010). Thinking aloud, en The Japan Times, 19.09.2010, http:/www.japantimes. co.jp/life/2010/09/19/to-be-sorted/thinking-aloud/\#.UbWXj-C9f3G

Pamment, J. (2014). Articulating influence: Toward a research agenda for interpreting the evaluation of soft power, public diplomacy and nation brands, Public Relations Review, 40, p.58.

Pappano, L. (2012). The year of the MOOC, en The New York Times, 02.10.2012, http:// www.nytimes.com/2012/11/04/education/edlife/massive-open-online-courses-are-multiplying-at-a-rapid-pace.html?smid=pl-

Sonenshine, T. (2014). Bottom Line Diplomacy Why Public Diplomacy Matters, 18-3-2014, http://www.state.gov/r/remarks/2013/210771.htm

Stengel, R. (2014). A message to the public diplomacy community, 18-3-2014, http://takefiveblog. org/2014/02/19/a-message-to-the-public-diplomacy-community-from-richard-stengel-under-secretary-for-public-diplomacy-and-public-affairs

Walberg, H. J. \& Thomas, S. C. (1972). Open Education: An Operational Definition and Validation in Great Britain and United States. American Educational Research Journal, 9(2), 197-208. (DOI: http://doi.org/czcqr6).

Whittemore, R. \& Knafl, K. (2005). The Integrative Review: Updated Methodology. Journal of Advanced Nursing, 52(5), 546-553. (DOI: http://doi.org/dhbpb8). 